\title{
Representaciones y medidas de la desigualdad. Una reflexión teórico-metodológica
}

\author{
César RENDUELES \\ Universidad Complutense Madrid, Sociología V \\ cesar.rendueles@cps.ucm.es \\ Igor SÁDABA \\ Universidad Complutense de Madrid, Sociología IV \\ igor.sadaba@cps.ucm.es
}

Recibido: 17-03-2014

Aceptado: 03-06-2014

\begin{abstract}
RESUMEN
La preocupación por los procesos de estratificación social es una de las claves de la arquitectura de las teorías sociológicas clásicas. Hoy disponemos de metodologías sofisticadas para el análisis empírico de la desigualdad, pero rara vez desembocan en explicaciones causales ambiciosas de la morfología social. La desigualdad desempeña un papel crucial como variable independiente en la explicación de una amplia gama de fenómenos sociales que van desde la experiencia subjetiva postmoderna hasta graves conflictos relacionados con la división internacional del trabajo. Este artículo analiza las características que deberían tener los indicadores de la desigualdad capaces de nutrir las teorías de largo alcance, indicando las limitaciones de algunos de los indicadores más utilizados al día de hoy y sacando a la luz algunas propuestas intuitivas de conceptualización y representación de la estratificación social. En concreto, se valorará la contraposición entre salarios y patrimonio, la escala temporal en la que se analizan los indicadores y se planteará la utilidad de algunos métodos de representación tales como el desfile de los salarios (Income parade).
\end{abstract}

Palabras clave: desigualdad, estratificación, medición, indicadores, clases sociales.

\section{Representations and measurements of inequality. Theoretical and methodological reflections}

\begin{abstract}
Concern for social stratification processes is one of the keys to the formation of classical sociological theories. Today there are sophisticated methodologies for empirical analysis of inequality, but rarely lead to ambitious causal explanations of social morphology. The inequality plays a crucial role as an independent variable in explaining a wide range of social phenomena ranging from post-modern subjective experience to serious conflicts with the international division of labour. This article discusses the features that should have indicators of inequality theories capable of nurturing long-range social theories, indicating the limitations of some of the indicators used today and bringing to light some intuitive proposals conceptualization and representation social stratification. In particular, the contrast between wages and heritage, the timescale in which the indicators are analyzed and the usefulness of some methods of representation such as the "Income Parade" will arise will be assessed.
\end{abstract}

Keywords: inequality, stratification, measurement, indicators, social classes. 


\section{REFERENCIA NORMALIZADA}

Rendueles, C. y Sádaba, I. (2014). "Representaciones y medidas de la desigualdad. Una reflexión teóricometodológica”. Cuadernos de Relaciones Laborales, Vol. 33, núm. 1, p.13-34.

SUMARIO: Introducción. 1. Teorías sin medida y medidas sin teoría. 2. Operacionalizaciones y medidas de la desigualdad. 3. Medidas intuitivas. 4. Conclusiones. 5. Bibliografía.

\section{Introducción}

Si alguien sin ningún conocimiento de la historia reciente de nuestro país examinara los principales indicadores de riesgo de exclusión y desigualdad del último cuarto de siglo e intentara inferir alguna información acerca de la dinámica socioeconómica española, seguramente llegaría a la conclusión de que se trata de un periodo de enorme estabilidad. Por ejemplo, según la web del INE, entre 2004 y 2012 -es decir, entre el pico del "milagro económico español” y el valle de una crisis económica brutal- la tasa de riesgo de pobreza o exclusión social varió de 24,4 a 26,8, es decir 2,4 puntos en ocho años ${ }^{1}$. A su vez, el Índice de Gini subió de 0,3395 en 1990 a 0,349 en 1995 para luego volver a bajar a 0,322 en 2008 y repuntar a 0,347 en 2010 (Fuente: OCDE e INE). Resulta llamativo que las dinámicas convulsas que caracterizan nuestra vida política, social y económica desde finales de la década de los ochenta tengan un reflejo tan amortiguado en estos instrumentos estadísticos.

Entre los procesos sociales que no han tenido ningún reflejo en los indicadores de desigualdad están: la desindustrialización y la tercerización del mercado de trabajo, los procesos de financiarización extrema y el endeudamiento generalizado de las familias vinculados a la burbuja inmobiliaria, la combinación de crecimiento del PIB y descenso de los salarios reales entre 1998 y 2008, la inmigración creciente y acelerada, los cambios en la estructura familiar, la transformación de la estructura fiscal, la incorporación de las mujeres al mercado de trabajo, la crisis de los cuidados o incluso la mayor crisis de la economía española de los últimos cincuenta años. Todo ello es completamente transparente para los indicadores de desigualdad al uso. ¿Cómo es posible?

Resulta inverosímil que estas transformaciones no hayan afectado profundamente a los procesos de estratificación social. De hecho, más bien parece razonable pensar lo contrario. En las dos últimas décadas, los cambios en el mercado laboral, la fiscalidad y las fuentes de beneficio de las élites han producido una creciente polarización social que se ha puesto de manifiesto en una gran cantidad de ámbitos y ha alterado las relaciones de poder político y social tradicionales (López y Rodríguez, 2011; Hernández Vigueras,_2012; Navarro, 2009; Fernández Durán, 2011; Garzón, 2013). Sin embargo, estos procesos sólidamente

${ }^{1}$ http://www.ine.es/jaxi/tabla.do?type=pcaxis\&path=/t25/p453/provi/10/\&file=01005.px 
teorizados y ampliamente intuitivos no han tenido una plasmación inteligible y directa en las herramientas de medición estadística habituales que utilizan las ciencias sociales.

La crisis económica ha sacado a la luz la magnitud de este problema, hasta el punto de que los debates metodológicos sobre cuestiones técnicas han llegado a ocupar las páginas de los diarios generalistas. Por ejemplo, "España es el país de la OCDE donde más crece la desigualdad” (El País, 15 de mayo de 2013) o “A más crisis, menos pobreza (relativa)" (Eldiario.es 1 de septiembre de 2013). En relación al segundo de estos artículos, la Encuesta de Condiciones de Vida de 2012 del INE informaba de que en 2012 había menos pobres en España que en 2011. Según sus estimaciones, la población que estaba por debajo del umbral de pobreza ${ }^{2}$ descendió 0,7 puntos respecto al año anterior (de $21,8 \%$ a $21,1 \%$ ). Por supuesto, esta mejoría resulta profundamente antiintuitiva y choca frontalmente con la idea de sentido común de que existe una relación directa entre la crisis económica y el empobrecimiento a causa del aumento del desempleo, el descenso de coberturas y prestaciones sociales o los recortes en distintos servicios públicos (Foessa 2013).

De hecho, los datos del INE fueron ampliamente cuestionados tanto por organizaciones sociales que tenían constancia de primera mano del aumento de la pobreza como por distintos centros de investigación no oficiales ${ }^{3}$. Las conclusiones del INE son el resultado de tres elementos encadenados y consecutivos que demuestran la fragilidad de las mediciones ${ }^{4}$ : i) los datos finales provienen de una medida general (para toda la población en su conjunto) pero la pobreza no es un proceso generalizado y varía según grupos de población (de hecho, para jóvenes y mayores de 65 años o para extranjeros no comunitarios la situación empeoró); ii) trabajamos con una medida relativa sensible a cambios generales (si toda la sociedad se empobrece, los umbrales de pobreza relativa bajan y algunas personas dejan de ser pobres a pesar de sus privaciones ${ }^{5}$ ), y iii) estamos ante un fenómeno episódico y la medida fotográfica y estática no describe adecuadamente procesos sociales de duraciones largas (los datos correspondían a 2011 pero en 2012 las tendencias se agravaron).

Sería injusto achacar este problema de interpretación de los datos a la

2 Según el propio INE: "La línea de pobreza o umbral de pobreza se fija en el 60\% de la mediana de la distribución de los ingresos por unidad de consumo adjudicados a las personas” (Estudio descriptivo de la pobreza en España, 2004).

${ }^{3}$ Véase "El gobierno comunica a Europa un dato increíble”, Público, 2 de mayo de 2013.

${ }^{4}$ Véase la explicación completa en: http://barometrosocial.es/por-que-disminuye-lapobreza-cuando-todo-parece-empeorar-entendiendo-las-estadisticas-oficiales/\#more-464

5 En este sentido, Chen y Ravallon (2013) realizan una crítica muy interesante de estos indicadores y sugieren la necesidad de una reconceptualización puesto que los tipos de pobreza relativa y absoluta están cambiando drásticamente en las últimas décadas. 
metodología del INE. En realidad, este organismo no hizo más que aplicar los criterios establecidos por la UE desde hace unos años. El nuevo indicador agregado AROPE (“At Risk Of Poverty and/or Exclusion”) fue incorporado en nuestro país por el INE por primera vez en 2010 en su Encuesta de Condiciones de Vida (anual). Según los términos de la propia UE, este indicador aglutina variables relativas a la “participación completa en la sociedad y el trabajo" (EAPN, 2012: 2).

El sistema AROPE se desarrolló con aspiraciones de universalidad e internacionalidad. Es un medidor diseñado para establecer comparaciones entre diferentes países a escala continental. Dado que las situaciones de desigualdad en Europa se ven condicionadas no solo por el nivel de renta sino también por la situación laboral, AROPE integra tres grandes factores: la renta, la privación material severa y la intensidad de trabajo. Desde luego, incluir el contexto laboral en las mediciones de la desigualdad es una iniciativa interesante y sugerente. Pero parece muy complicado examinar las diferentes situaciones nacionales mediante conceptos sociológicamente poco informativos como la "intensidad laboral" ${ }^{6}$. El objetivo de incrementar la comparatividad transnacional obliga a recurrir a términos ambiguos despreciando aspectos cruciales de las relaciones laborales muy diferentes en los distintos países europeos: los niveles de presión laboral, la precariedad por temporalidad, el poder contractual de trabajadores y empresarios, los sectores laborales predominantes o las condiciones laborales. La ampliación del campo conceptual de la pobreza y la exclusión se compensa con una baja dependencia explicativa. De hecho, sabemos que el índice compuesto AROPE ha pasado en nuestro país del 23,1\% en 2008 al 26,7\% en 2011 (elaboración de CEET a partir de la Encuesta de Condiciones de Vida (2008-2010) del $\mathrm{INE}^{7}$ ) pero carecemos de una visión completa de cómo o por qué ha sucedido.

Estos ejemplos ponen de manifiesto, por un lado, la baja eficacia teórica de algunas medidas técnicamente sofisticadas de la desigualdad, cuya traducción al lenguaje sociológico apenas refleja cuestiones sustanciales. Y, por otro, la dificultad de operacionalizar un concepto central de las ciencias sociales, hoy en día de trágica actualidad y que arrastra una "polémica inacabada" (Escobar, 1998: 9).

\footnotetext{
${ }^{6}$ Definida como "la relación entre el número de personas que trabajan en un hogar y el de las que están en edad de trabajar. A modo de ejemplo podría decirse que, en un hogar con dos adultos en el que sólo trabaja uno a jornada completa, la intensidad del trabajo es de 0,5, mientras que si trabajan los dos, la intensidad del trabajo es de 1; si uno de los adultos trabajara a media jornada y el otro a jornada completa, la intensidad de trabajo de este hogar es de 0,75. El indicador comprende a las personas que viven en hogares con una intensidad de empleo inferior al 0,2.” (EAPN, 2012: 3).

7 European AntiPoverty Network:

http://www.accem.es/ficheros/documentos/pdf_varios/DOC_FINAL_Nuevas_propuestas_ para_nuevos_tiempos_def.pdf
} 


\section{Teorías sin medidas y medidas sin teorías}

El interés por la evaluación de la magnitud e intensidad de la desigualdad social fue un elemento esencial de la arquitectura de las teorías sociológicas clásicas. Los orígenes sociales del capitalismo industrial están íntimamente asociados al problema urbano del pauperismo, una nueva forma de estratificación profunda que la teoría sociológica decimonónica trató de explicar y, al menos programáticamente, medir (Dubet 2006 y Álvarez-Uría y Varela, 2011).

Los fundadores de las ciencias sociales modernas intentaron entender un tipo de estratificación al mismo tiempo extremo y compatible con altos estándares de igualdad jurídica y, en consecuencia, causalmente poco intuitivo. En ese sentido, la época heroica de la teoría social produjo excelentes reflexiones generalistas sobre el papel de las desigualdades pero, a causa de las limitaciones metodológicas, escasas o no muy fiables colecciones de datos. La sociología clásica se caracterizó, en ese sentido, por un hiperdesarrollo del análisis teórico de la desigualdad y un subdesarrollo del análisis empírico. La pobreza era crudamente manifiesta en las calles de las urbes europeas y se percibía e intuía su relación con la extensión fabril y la implantación del modelo mercantil liberal. Pero no existían instituciones dedicadas a la producción de datos primarios o secundarios, registros socioeconómicos ni instrumental metodológico fiable que permitiera una verificación directa de todo ello.

Hoy, en cambio, disponemos de múltiples organismos locales, nacionales e internacionales casi únicamente dedicados a producir datos estadísticos (encuestas, padrones y censos, datos sociodemográficos y económicos, etc.) adecuados para la contabilidad sociológica y política y, en concreto, para producir medidas precisas de los procesos de desigualdad que atraviesan las sociedades contemporáneas. Existen centros de investigación, institutos de estadística, oficinas europeas, organismos mundiales, entes privados, investigadores académicos, etc. en actividad frenética para generar material de análisis actualizado.

Sin embargo, el desarrollo formal de herramientas metodológicas de medición de la desigualdad rara vez ha sido el correlato de explicaciones causales ambiciosas e intuitivas (Blalock, 1991, Brady, 2003 y Cowell, 2009). Las mediciones conducen a interpretaciones de la estratificación o bien fragmentarias y muy técnicas o bien explicativamente romas e insatisfactorias. Más en concreto, desde las ciencias sociales, auténticos centros de interpretación de los procesos de desigualdad, hemos tendido a delegar las tareas de medición en disciplinas colindantes más técnicas (estadística, matemática, economía, etc.) debido a ciertas incapacidades instrumentales o a la ausencia de herramientas propias. A pesar de muchos esfuerzos multidisciplinares, los resultados de esta transferencia han sido algo irregulares: pulcros modelos matemáticos sin traducción directa a la empiria social o un collage apañado de medidas aproximadas muy interpretables pero sin fuentes de datos o con propiedades indescifrables. Es más, se ha tendido a vaciar de contenido o incluso a prescindir del repertorio conceptual -como las nociones de clase social o de explotación- que la tradición sociológica elaboró para abordar 
procesos de estratificación históricamente muy frondosos. La desigualdad no es un fenómeno meteorológico, sino una dinámica social con ganadores y perdedores que es el resultado de un juego de estrategias políticas complejo y ambiguo. Numerosos indicadores de la desigualdad no sólo no proporcionan información empírica útil para esta clase de análisis, sino que más bien los obturan. Las ciencias sociales tienen la asignatura pendiente de encontrar instrumentos que les permitan contabilizar y catalogar con cierta precisión las desigualdades sociales sin perderse en laberintos matemáticos o axiomas crípticos y, a la vez, ser capaz de integrar tales medidas en modelos teóricos históricos concretos ${ }^{8}$.

Los nefastos efectos sociales de la las políticas económicas neoliberales provocaron un renovado interés por las medidas empíricas de la desigualdad en las décadas de 1980 y1990 (Sen y Foster, 1996) que supuso una amplia revisión del marco teórico precedente (Atkinson, 1970). Del mismo modo, la situación actual de nuestro país y de las regiones circundantes ha incrementado mucho los esfuerzos por evaluar la dinámica de las desigualdades sociales actuales ${ }^{9}$.

Este retorno de la preocupación por la medición de la estratificación tiene características diferenciales respecto a la sociología clásica y moderna. Hoy se hace énfasis en lo global, se buscan comparaciones de más larga duración y se intentan superar tanto los indicadores macroeconómicos simples (el PIB per cápita, por poner un ejemplo) como las medidas exclusivamente monetarias (Heshmati, 2004). Del mismo modo, los estudios recientes tienden a incidir en la imbricación generalizada de las desigualdades con toda una serie de fenómenos aparentemente dispersos e inconexos, como la salud mental o la autoestima (Wilkinson y Pickett, 2009). Estos cambios son sintomáticos de la insatisfacción entre un amplio grupo de científicos sociales con la concepción heredada de la relación entre medición y explicación de la desigualdad social y nos abocan a revisar críticamente la utilidad de los instrumentos estadísticos disponibles para renovarlos si fuera necesario.

${ }^{8}$ Cabe mencionar que los esfuerzos por superar esta compartimentalización del estudio de la desigualdad se han producido sin cesar. Un ejemplo son el LIS Datacenter (Bases de datos LIS-Luxemburgo, http://www.lisdatacenter.org/our-data/lis-database/), Euromod (Essex, https://www.iser.essex.ac.uk/euromod) o algunos trabajos de Eurostat en los que ha tratado de encajar todas las piezas del puzzle de la desigualdad, conjugando precisión empírica con ciertos marcos teóricos interdisciplinares.

9 “España es el país con mayor desigualdad social de la eurozona” (El País, Economía, 11 octubre 2012). 


\section{Operacionalizaciones y medidas de desigualdad}

Por supuesto, es un lugar común que la operacionalización, la construcción de datos, exige que el investigador tome decisiones prácticas contingentes. Sin embargo, al desarrollar indicadores de desigualdad mediante técnicas estadísticas elaboradas a menudo se olvida esta sencilla verdad. La elección concreta de una definición operativa que permita asociar conceptos teórico-abstractos a elementos observables empíricamente que cuantifiquen las asimetrías sociales no resulta nada sencilla.

El empirismo ingenuo parece suponer que los números están desperdigados libremente por el mundo y que basta con recolectarlos cuidadosamente y proceder a su descifrado. Lo cierto es que establecer protocolos para una correcta medición -es decir, asociar el fenómeno a medir con indicadores concretos- consiste en una serie de elecciones sucesivas cuyas consecuencias desbordan la metodología entendida en un sentido restrictivo. Las variables que optamos por visibilizar, sus tasas y sus representaciones gráficas, las escalas o los intervalos de tiempo e incluso las poblaciones y geografías que observamos, determinan en gran medida nuestras percepciones, validando o falsando hipótesis según la combinación de estos parámetros ${ }^{10}$.

Existen numerosos indicadores o variables para medir la desigualdad, cada uno de ellos con propiedades, virtudes y defectos. En principio, su utilidad depende de las exigencias formales que planteemos y del rendimiento empírico que esperemos de ellos, por no mencionar de la relación que tengan con nuestras presuposiciones teóricas y la disponibilidad de datos. Son cuestiones cruciales que a menudo, se descuidan.

Los requisitos matemáticos y formales generalmente admitidos para los indicadores de la desigualdad son: la independencia de escala, la independencia del tamaño poblacional, la independencia ante cambios de posición, el principio débil de transferencias (de hogar rico a pobre), el principio fuerte de transferencias (de hogar rico a pobre y proporcional a su distancia), la descomposición aditiva y el rango del índice. Podríamos resumir todos ellos en tres grandes ideas (CEPAL, 2001: 20-21): la independencia de escala o de media, la independencia del tamaño poblacional y la condición de Pigou-Dalton (principios de transferencia).

Estas condiciones presuponen un cierto isomorfismo entre el mundo real y el

10 "La investigación empírica es un medio de obtener respuestas sobre la realidad. Nuestras preguntas pueden ser esencialmente prácticas o de interés principalmente académico. En cualquiera de los casos, lo probable es que las formulemos en términos abstractos. Y, sin embargo, las respuestas que deseamos suelen ser concretas y específicas. Uno de los primeros problemas de la investigación es el de idear el modo de obtener, partiendo del nivel abstracto de las preguntas, algunas observaciones concretas que nos permiten responder a ellas.” (Manheim y Rich, 1988: 67). 
orden matemático. Se trata de requisitos estadísticos y analíticos, pero se espera que, además, reflejen la estructura real de las relaciones sociales. Es decir, aunque se plantean por razones formales deben tener un correlato en las situaciones empíricas. Por ejemplo, si se aplica una medida redistributiva que transfiere mediante impuestos cierto nivel de renta desde un hogar rico a un hogar pobre se espera que esto tenga algún efecto en la medida de la desigualdad social (principio de transferencias). También, resulta intuitivo pensar que la desigualdad social de un país debería ser la suma o el promedio de las desigualdades observadas en sus provincias o comunidades (descomposición aditiva). Dicho de otro modo, al menos como ideal normativo existe un vínculo entre los paradigmas teóricos y las medidas empíricas. Las características de estas últimas no derivan únicamente de ciertas condiciones matemáticas sino también de la búsqueda de interpretaciones sociológicamente coherentes.

Tomando como criterio de clasificación tanto los requisitos formales como el resultado empírico esperado, podemos distinguir cuatro grandes familias de indicadores en la historia reciente de estudios sobre desigualdad (McKay, 2002), cada una de ellas con su propio conjunto de problemas y limitaciones.

A) La primera familia se podría asociar a las medidas estadísticas, tales como las medidas de dispersión (coeficiente de variación de Pearson, varianza, desviación típica o estándar, etc.) que son sencillas de calcular y relativamente intuitivas. Asimismo, esta familia de estadísticos permite describir individuos con respecto a la población en la que se encuentran y medir la diversidad (Escobar, 1998). Sin embargo, como es bien sabido, este tipo de medidas no satisfacen algunos requisitos formales o teóricos importantes, como la independencia de escala, a causa de su fuerte dependencia de los valores medios. Por ejemplo, la varianza se calcula siempre como la media de las distancias a la media aritmética (al cuadrado) y dependen mucho de la escala, las unidades o los valores medios (tiene carácter de magnitud absoluta y se mide en unidades cuadráticas, Escobar, 1998: 12). Igualmente no respetan el criterio de rango del índice al no estar confinados o limitados a pequeños intervalos (0-1), pudiendo dar valores excesivos en ciertas circunstancias (desviaciones típicas mayores que la media, por ejemplo). Aunque la solución pasa por utilizar medidas relativas (coeficiente de variación) también nos podemos encontrar con resultados no deseados cuando la variable tiene valores negativos o la media es próxima a cero (Escobar, 1998: 14).

B) La segunda familia se deriva de la curva de Lorenz, que representa el porcentaje acumulado de una variable (ingresos) por un determinado grupo de población (porcentaje de población) (Heshmati, 2004:1-2). El índice de Gini, basado a su vez en la curva de Lorenz, mide hasta qué punto la distribución del ingreso (o, en algunos casos, el gasto de consumo) entre individuos u hogares dentro de una economía se aleja de una distribución perfectamente equitativa. El coeficiente de Gini se basa en una representación gráfica de una función de distribución acumulada y se define matemáticamente como la proporción 
acumulada de los ingresos totales (eje y), que obtienen las proporciones acumuladas de la población (eje x). Durante mucho tiempo, el índice de Gini ha sido el medidor oficial, estándar y universal de las desigualdades de renta y, por ende, de las desigualdades sociales. Sin embargo, la formalidad del índice, basado en un modelo de distribución de percentiles de renta y población, tiene también sus problemas.

Una de las principales limitaciones del índice de Gini es su insensibilidad a cambios en la distribución que deja inalterada la curva por debajo de los 45 grados. Además, no cumple el axioma fuerte de transferencias, plantea resultados ambiguos con curvas cruzadas (hasta el punto de que en países muy pobres llega a dar riqueza neta negativa) y, finalmente, no cumple la propiedad de descomposición aditiva ${ }^{11}$. Pero, al margen de estos problemas técnicos, el índice de Gini tiene problemas relacionados con su rendimiento empírico. Por ejemplo, en pequeñas escalas no proporciona valores muy diferentes en contextos geográficos completamente diversos. En 2010, las diferencias en el índice de Gini entre países del sur de Europa como España $(33,9)$ y Portugal $(33,7)$ y otros del norte como Dinamarca $(26,9)$ y Finlandia $(25,4)$ eran de menos de 10 puntos sobre 100 (realmente no más de 8 puntos), cuando la percepción social es que son países cuyas situaciones de desigualdad están muy alejadas ${ }^{12}$. La media de EU-27 está en 30,5 pero es que el rango o recorrido europeo es muy estrecho, casi todos los países continentales se sitúan en un margen reducido (entre 23 y 35, aproximadamente) lo que no parece proporcionar una visión ajustada de las altas disparidades entre algunas regiones y otras y menos aún de las causas de esas diferencias. En España, por otra parte, si uno midiera el valor absoluto del índice de Gini en $1999(32,0)$ y en $2010(33,9)$, apenas podría constatar alguna variación de las desigualdades en estos once años (una subida de 2 puntos sobre 100), cuando sabemos que han tenido lugar transformaciones drástricas. Lo que ha ocurrido es que este coeficiente ha experimentado ciertas oscilaciones que, en términos netos, no refleja grandes cambios sociales. Igualmente, se considera que la curva de Lorenz es muy sensible a pequeños errores en la recogida de datos debido a su poca robustez (Heshmati, 2004: 2).

C) Existe un conjunto de indicadores basados en funciones de utilidad y entropía desarrollados por teóricos de la economía, como el índice Dalton, el índice Atkinson, el índice de Theil o el índice generalizado de entropía (procedente de la teoría matemática de la información). Son índices normativos que emplean

11 "El coeficiente de Gini tiene mal ajuste cuando los valores de las variables son negativos. Además, tiene la misma propiedad que el coeficiente de variación de Pearson: al multiplicar por una constante los valores de la variable, se mantiene inalterado el coeficiente; pero no sucede lo mismo si se le añade una cantidad.” (Escobar, 1998: 24).

12 Datos procedentes de Eurostat:

http://epp.eurostat.ec.europa.eu/tgm/table.do?tab=table\&language=en\&pcode=tessi190 
formulas complejas y cuya construcción matemática es muy elaborada (Atkinson, 1970). Estos indicadores plantean amplias garantías formales pero tienen una utilidad cuestionable en las mediciones empíricas reales y en su interpretación. Por ejemplo, el índice de Atkinson, tal vez el más conocido por haberse aplicado al índice de desarrollo humano ajustado por desigualdad, trata de cumplir tres requisitos que lo hacen preferible al índice de Gini.

(i) Consistencia de los subgrupos, de modo que si la desigualdad desciende en un subgrupo (región, grupo étnico, etc.) y permanece invariable en el resto de la población, la desigualdad general también desciende.

(ii) Énfasis en la desigualdad en el extremo más bajo de la distribución. Por su construcción, el coeficiente de Gini otorga el mismo peso a toda la distribución, mientras que el índice de Atkinson hace más hincapié en el extremo inferior, por lo que refleja mejor la mortalidad infantil, el analfabetismo y la pobreza de ingresos.

(iii) Sencillez de cálculo y elegancia matemática.

El problema de esta tercera familia de indicadores deriva de la artificialidad de su concepción. Son medidas cocinadas en laboratorios estadísticos o académicos y diseñadas a fuego lento por expertos teóricos. El resultado son pulcros esquemas de composición minuciosa de indicadores y de exquisitas fórmulas matemáticas. Pero la ausencia de datos empíricos suficientes y de repositorios completos para diversas regiones o países, así como la complejidad de los cálculos a realizar, acaba dando lugar a algoritmos decorativos inaplicables.

Por ejemplo, el índice de Atkinson, utilizado en las correcciones al IDH, se considera un índice normativo basado en funciones de bienestar. Estos índices se basan en el uso de funciones de bienestar social que reflejan los juicios de valor implícitos acerca de la relación entre desigualdad y bienestar. El índice de Atkinson mide la fracción de renta que puede ser sacrificada sin pérdida de bienestar social si la renta fuese distribuida igualitariamente. Pero la obtención de datos sobre lo que es bienestar social es tan compleja que es necesario utilizar bases de datos internacionales (Duclos y Araar, 2006) que trabajan únicamente con rentas y salarios oficiales y donde el bienestar queda definido a partir de otro conjunto extenso de variables muchas veces no explícitas o visibles o que se obtienen de fuentes diversas (Urban, 2013) ${ }^{13}$.

13 De hecho, NNUU, en los cálculos de IDH ajustado por desigualdad dicen: "La principal desventaja del IDH-D es su insensibilidad a la asociación, es decir, que no captura las desigualdades simultáneas. Para que la medida sea sensible a este aspecto, la totalidad de los datos de cada individuo debe provenir de una única encuesta, lo que actualmente es imposible 
D) Por último, existe una familia de indicadores sociológicos relacionados con la pobreza y el desarrollo. El índice de desarrollo humano (IDH) es seguramente el más conocido y constituye un esfuerzo interesante por superar las medidas meramente econométricas integrando otras dimensiones sociales del desarrollo. Igualmente, Naciones Unidas y su entorno institucional llevan unos años aplicando indicadores compuestos para cuantificar la pobreza en cada país.

El problema es que estos indicadores que miden la pobreza absoluta a partir de un estándar universal se componen a su vez de varios subindicadores cuya elaboración requeriría datos de todos los países del mundo. Estos indicadores se enfrentan al reto de calibrar la pobreza en las distintas latitudes superando las enormes disparidades que existen entre unos lugares y otros. Dicho de otro modo, el indicador creado para comparar la pobreza a nivel global se enfrenta a la limitación de que es imposible comparar la pobreza a nivel global. Así que, de una forma ligeramente caricaturesca, Naciones Unidas creó dos indicadores distintos con la intención de aplicar uno a países pobres o en vías de desarrollo y otro a países ricos o desarrollados (los IPH1 e IPH2). Para solventar la asimetría entre esos dos indicadores y disponer de una medida estándar mundial fabricó posteriormente un índice sintético universal, el IPM (Índice de Pobreza Multidimensional ${ }^{14}$ ) que se ha convertido en el patrón general pero cuyo uso es relativamente poco habitual (UU NN, 2010; Milanovic, 2012). En un formato relativamente afín, Sabina Alkire y James Foster implementaron un método de medición multidimensional de la pobreza ${ }^{15}$ que ha sido usada por alguna institución como el Iniciativa de Desarrollo Humano y Reducción de la Pobreza (OPHI).

Un intento de superación adicional de algunas de estas limitaciones han sido los así llamados indicadores de privación tales como el índice de Towsend ${ }^{16} \mathrm{y}$ otros similares ${ }^{17}$. El estudio de la privación multidimensional se remonta a los años 1980, cuando se esbozaron los primeros intentos de analizar la pobreza y la exclusión social a través de indicadores no monetarios. Destacan, en estos años, las propuestas precoces de Peter Townsend en 1979 (y, posteriormente, otros autores como Mack y Lansley, Callan, Nolan y Whelan). Townsend, investigador de la Universidad de Bristol y de la LSE además de asesor de UNICEF, en concreto, construyó un índice de privación multidimensional partiendo de sesenta indicadores que reflejaban las condiciones de vida (alimentación, ropa, salud/sanidad, ocio, equipamiento de la vivienda y recursos, posesión y acceso a bienes duraderos, etc.). Lo que Townsend buscaba con este indicador agregado de privación multidimensional era estudiar si

cuando se trata de un grupo grande de países.” (Informe Desarrollo Humano 2013, notas técnicas, http://hdr.undp.org/en/media/HDR\%202013\%20technical\%20notes\%20ES.pdf).

14 http://hdr.undp.org/es/estadisticas/ipm/

${ }^{15}$ http://www.ophi.org.uk/research/multidimensional-poverty/alkire-foster-method/

${ }^{16}$ http://en.wikipedia.org/wiki/Townsend_Deprivation_Index

${ }^{17}$ http://www.poverty.ac.uk/definitions-poverty/deprivation-and-poverty 
existía un nivel de ingresos a partir del cual el número de privaciones aumentaba escandalosamente, es decir las condiciones de vida empeoraban drásticamente (Towsend, Phillimore and Beatie, 1988) para poder tomar este nivel de renta como verdadero umbral de la pobreza ${ }^{18}$. Es decir, se introducía la idea de que las definiciones de pobreza o de desigualdad dependían en cada momento y lugar del gradiente de acceso a ciertos bienes definidos a priori como básicos o necesarios para la vida "normal”. Hay quien considera que Towsend pretendía rebatir la noción de pobreza absoluta derivada de los trabajos de Amartya Sen y otros autores.

Finalmente, cabe mencionar los esfuerzos de Piketty y Saez por facilitar un registro de la distribución de renta basado en la concentración de riqueza (top incomes). El objetivo principal de estos autores era construir una base de datos internacional de alta calidad y largo recorrido sobre renta y concentración de riqueza usando estadísticas históricas de impuestos. La base resultante incluye series que cubren gran parte del siglo XX para muchos de los países occidentales (Piketty y Saez, 2006).

Así, estas últimas familias de indicadores son las que permiten una lectura más fructíferamente sociológica y cercana a las teorías de la estratificación. El problema es que o bien carecen de datos fiables o no cumplen siempre los requisitos formales o se limitan a lidiar con situaciones o casos no comparables. De hecho, el resultado natural de las compilaciones realizadas por organismos internacionales con este tipo de indicadores no deja de ser tablas extensas con muchos países o mapas (cartogramas) de colores variados pero poca interpretación histórico-social. El resto de "medidores", a veces fabricados y diseñados a través de catálogos extensos de variables o indicadores particulares no siempre se pueden abastecer de información completa para todos ellos o proporcionan resultados complejos e incluso crípticos de interpretar.

\section{Medidas intuitivas}

La reivindicación de mediciones de la desigualdad informadas por teorías de la estratificación con ambiciones explicativas está sujeta a una objeción sencilla pero aguda. Parece absurdo y pernicioso forzar los indicadores para que muestren lo que nuestras teorías esperan. Una respuesta igualmente sencilla es que, de hecho, eso es lo que siempre sucede en alguna medida. Es llamativo que numerosos cambios en el instrumental empírico de análisis de la desigualdad obedecen no a criterios técnicos sino a su inadecuación a las intuiciones causales. La dualidad entre el IPH 1 y el IPH2, por ejemplo, es muy coherente con las teorías de la dependencia acerca del desarrollo desigual del centro de la economía mundial y su periferia (Wallerstein, 2001; Chossudovsky, 2002). Los indicadores que intenten superar formalmente esta

18 Tomado de La pobreza y su medición. Presentación de diversos métodos de obtención de medidas de pobreza (INE, http://www.ine.es/daco/daco42/sociales/pobreza.pdf). 
asimetría -como el IPM - tienen un bajo rendimiento empírico porque la diferencia entre los dos IPH es en sí misma informativa aunque incómoda de manejar en algunas situaciones.

Aunque es difícil generalizar, muchos índices se refinan para que den resultados cercanos a la comprensión no operacionalizada de los investigadores acerca de la naturaleza de la desigualdad en las sociedades contemporáneas. Así ocurrió, por ejemplo, con el IDH, cuyos criterios de elaboración variaron cuando algunos países árabes con rentas elevadas (PIB per cápita dependientes de petróleo, por ejemplo) empezaron a puntuar "excesivamente" alto en desarrollo humano cuando el resto de factores (educación y sanidad) se mantenían constantes. Para ello, hubo que sustituir la media aritmética de los índices de economía, educación y sanidad (fácilmente alterable por uno solo de los factores) por una media geométrica (que solo registra cambios cuando varían todos los factores). Esta situación, científicamente insólita, es un síntoma de la dependencia de las mediciones de la desigualdad de precomprensiones amplias que, en todo caso, deben ser explicitadas.

Dicho de otro modo, más allá de los experimentos puramente formales, los criterios de medición empírica siempre se adaptan al terreno y se van modificando cuando los resultados obtenidos son escandalosamente poco intuitivos. Si las cifras chocan frontalmente con nuestros modelos sociológicos o nuestras percepciones generales se suelen revisar las herramientas de registro y cuantificación (como resumía un viejo chiste: "si los datos no concuerdan con la realidad, peor para los datos"). Es importante, por consiguiente, que ese proceso de "cocinado" se explicite teóricamente estableciendo su relación con esquemas explicativos históricamente fundados.

Sin embargo, la tendencia dominante entre muchas agencias encargadas de suministrar esta información discurre más bien en sentido contrario. A menudo se intenta oscurecer esa limitación constituiva de este campo de estudio recurriendo a formulaciones matemáticas complejas de rendimiento empírico endeble. De esta forma, las asimetrías sociales se muestran como algo dado e incontestable. Cuando los datos contradicen la observación cotidiana o las comprensiones teóricas informadas por fuentes estadísticamente poco sofisticadas, se tiende a adaptar las explicaciones a los resultados formalmente más refinados. El resultado es que la desconexión entre la base teórico-sociológica y la información empírica genera un desajuste que normaliza situaciones históricas de conflicto de intereses como si fueran estadios naturales.

En ese sentido, planteamos tres propuestas:

A) Sería importante recuperar un concepto amplio e intuitivo de las clases sociales -basado en la renta y el patrimonio, la capacidad de control sobre el trabajo y el estatus social- que, sin ánimo de exhaustividad o precisión formal extrema, se postule como un eje explicativo básico que permita relacionar las distintas situaciones de desigualdad y de sentido a las mediciones. De esta manera, con una noción completa y flexible de la idea de clase, no se perdería el horizonte explicativo que sustenta los indicadores empíricos y es posible realizar 
operacionalizaciones coherentes e interpretables (véase, por ejemplo, el análisis cauteloso de Escobar para diferentes medidas de desigualdad educativa e ideología, 1998: 28-35).

En ese sentido, por ejemplo, en España se ha tendido a infravalorar sistemáticamente el papel de las rentas patrimoniales en los procesos de estratificación. Durante muchos años, el empobrecimiento ha sido invisible porque estaba vinculado a procesos económicos generales relacionados con la acumulación de capital a través de activos inmobiliarios y financieros que ha tenido efectos brutalmente dispares entre las élites enriquecidas y las clases populares, que han quedado estructuralmente endeudadas (López y Rodríguez, 2001). España es un país muy patrimonialista, con una alta tasa de casas y pisos en propiedad frente a la predominancia del alquiler en otros países europeos, y donde las herencias familiares han jugado un notable papel en la trasmisión de estatus y clase. Pero, igualmente, es un país profundamente endeudado a causa de la generalización de préstamos hipotecarios a muy largo plazo (Del Río, 2008). Las medidas empíricas basadas en la renta deberían tener en cuenta tanto esta situación de endeudamiento, corrigiendo las rentas a la baja, como otro tipo de indicadores complementarios: consumo, desempleo, desahucios, posesiones, etc.

Eso permitiría también medir el enriquecimiento de las élites como un proceso directamente relacionado con el empobrecimiento de las clases populares. El último informe de FOESSA titulado Desigualdad y derechos Sociales alertaba que la diferencia entre las rentas más altas y las más bajas se ha incrementado un $30 \%$ desde 2006 (FOESSA, 2013). Pero el dato más llamativo del informe era el crecimiento de las rentas muy altas, algo de lo que se hicieron eco algunos medios $^{19}$. En ese sentido, la crisis económica no solo ha dejado un reguero de pobreza o exclusión sino un boyante mercado del lujo en plena expansión. Tal y como informaba el diario El Economista el 1 de abril del 2011 con respecto a uno de los bienes de consumo más habituales: "Las ventas de coches caen un 27\% y en el segmento de lujo crecen un 144\%”. Aunque hay pocos estudios oficiales, numerosas consultoras y empresas privadas constatan la inflación de marcas ostentosas y lujosas a la vez que la crisis económica se instalaba en nuestro país: "El lujo 'made in Spain' sortea la crisis con un aumento de las ventas del 15\%" ( $A B C, 25$ de diciembre de 2012, citando un estudio de la asociación Luxury Spain $^{20}$. Las desigualdades no solo se observan en los escalones inferiores de la

19 “En España los más ricos ganan siete veces más que los más pobres” (Público, 21 de marzo de 2013).

${ }^{20}$ Las noticias sobre el tema abundan aunque suelen pasar inadvertidas: "Las viviendas de lujo se siguen despachando en plena crisis” (El Mundo, 21 de febrero de2013), "El sector del lujo esquiva la crisis y prevé crear hasta 30.000 empleos en España hasta 2020” (Europa Press, 10 de diciembre de 2012), etc. Según el Observatorio del Mercado Premium y de Productos de Prestigio de IE Business School y Master-Card en España existe un mercado 
estructura social sino en el movimiento general que va de unas posiciones a otras o en la emergencia de opulentas y acaudaladas élites globales (Freeland, 2012).

Sin embargo, no se trata sólo de una rectificación técnica. Necesitamos indicadores que relacionen la desigualdad efectiva con los procesos que la generan (en este caso, el modelo de creación de riqueza en España). Y para eso es imprescindible ampliar el repertorio de instrumentos de medición. De modo análogo, Bourdieu se dio a conocer en la escena sociológica por utilizar el consumo cultural como distintivo de clase, una aportación que le permitió identificar fenómenos de estratificación y distinción social en la sociedad francesa de la segunda mitad del siglo XX a través de indicadores hasta la fecha ninguneados. Resulta perentorio desarrollar mecanismos de examen sociológico que no traten el empobrecimiento como un hecho aislado sino como parte de procesos históricos conflictivos y de largo recorrido.

Por ejemplo, el último Censo de Población y Viviendas del INE del 2011, cuya nota de prensa se publicaba este 18 de abril de 2013, incluye el dato de que en los últimos 10 años el número de viviendas vacías ha aumentado en 336.943 casos, un 10,8\% más. Es decir, si en 10 años y dado el aumento general de singles (hogares de un solo miembro) hay un $11 \%$ de viviendas libres, eso parece significar una "apelotonamiento" o agrupamiento familiar en torno a ciertas viviendas. Es un dato aparentemente anecdótico que, sin embargo, pone de manifiesto los efectos sobre la desigualdad social de la especulación inmobiliaria que fue el motor del crecimiento económico español durante la "década dorada" de 1998-2008.

En la misma línea, el colectivo IOE ha propuesto otras medidas que reflejen la situación de deterioro socioeconómico de las familias españolas tales como el porcentaje de ellas que han reducido sus vacaciones a solo una semana al año, las que deben afrontar gastos imprevistos, las que comienzan a tener muchas dificultades para llegar a fin de mes o las que tienen problemas para afrontar los pagos relacionados con la vivienda principal (hipotecas, alquileres, etc.) ${ }^{21}$.

Igualmente, Wilkinson y Pickett (2009) han estudiado los fenómenos de desigualdad atendiendo a sus consecuencias o efectos sociales más nocivos y recientes: tasa de población encarcelada, tasa de embarazo adolescente, desconfianza hacia los demás, esperanza de vida, rendimiento académico, violencia, obesidad, etc. No se trata de verdaderos indicadores de desigualdad pero, en la medida en que se trata de un fenómeno complejo y multidimensional, podemos acercarnos a él a través de una plétora de variables altamente correlacionadas. Wilkinson y Pickett miden estas correlaciones a partir de diagramas de dispersión y del coeficiente de correlación lineal de Pearson, que ponen de manifiesto relaciones lineales entre la desigualdad y este tipo de problemáticas en países e incluso

potencial para los productos de lujo personal estimado en el 20\% de las unidades familiares.

${ }^{21}$ IOE (Barómetro Social de España): http://barometrosocial.es/por-que-disminuye-lapobreza-cuando-todo-parece-empeorar-entendiendo-las-estadisticas-oficiales/\#more-464 
provincias.

La moraleja es que la medición empírica de la desigualdad no pasa necesariamente por adscribir un dígito tremendamente preciso a la situación de un país o una región. A menudo resulta mucho más provechoso identificar variables afines y fenómenos circundantes que podemos poner en relación con un marco teórico que aspira a explicar las dinámicas históricas de estratificación.

B) Las escalas temporales para el análisis de la desigualdad deberían estar históricamente fundamentadas y mediadas por decisiones de orden teórico. Por ejemplo, el índice de Gini y otros indicadores de desigualdad económica se computan sólo para un año y no capturan lo que está ocurriendo a lo largo del tiempo ni los ciclos para ciertas sociedades. De hecho, “dos sociedades con exactamente el mismo coeficiente de Gini podrían ser completamente distintas en términos de movilidad, oportunidades individuales, vulnerabilidad y diferencias intergeneracionales a lo largo del tiempo” (Graham, 2002).

En la misma línea y, sin despreciar los indicadores fotográficos o estáticos, puede resultar conveniente distinguir, al menos formalmente, medidas a corto y medidas a largo plazo en términos empíricos, especialmente cuando se evalúan intervenciones, programas de instituciones o políticas públicas (Vakis y Schdmit, 2010). No en vano, muchas de las técnicas de representación gráfica actuales hacen uso de las series temporales para ilustrar las variaciones con grandes escalas de tiempo (es el caso, por ejemplo, del Gapminder).

La decisión acerca del marco temporal significativo está determinada por elecciones conceptuales vinculadas a una comprensión general de cuáles son los procesos de estratificación que están teniendo lugar y cuál es su causa. Siguiendo con el ejemplo anterior, una de las razones del fracaso de las agencias oficiales españolas a la hora de informar de los procesos de polarización económica que han dado lugar a la crisis actual ha sido su desatención sistemática a cambios históricos en los procesos de acumulación de capital desde principios de los años ochenta: la destrucción del tejido industrial relacionada con el ingreso de España en la UE y los cambios en el mercado laboral que produjo, la transición fiscal que transformó la estructura impositiva haciéndola reposar sobre las rentas del trabajo, la especulación inmobiliaria como motor económico e incluso un modelo político diseñado para potenciar la complicidad de élites políticas y financieras. Todo ello es completamente indetectable al margen de un marco temporal amplio pero bien definido, vinculado a hipótesis históricas acerca de la periodización y el desarrollo de estos cambios que, en el caso de España, se remontan al tardofranquismo.

Un buen ejemplo de las limitaciones de los enfoques formalistas es la convención de que solo se considera que un país entra en recesión cuando durante dos o tres trimestres consecutivos el PIB crece negativamente. E igualmente, con independencia de cualquier otro proceso o situación adicional, las crisis macroeconómicas terminan oficialmente cuando dicho indicador agregado nacional pasa a saldo positivo. No deja de producir, en términos weberianos, un cierto extrañamiento ante un sistema de indicadores opaco e impersonal que nos informa 
de las cesuras como si fueran transiciones instantáneas y externas.

C) La decisión sobre las herramientas adecuadas para visualizar un proceso de desigualdad social es una cuestión metodológicamente crucial (Cowell, 2009:1736). En términos generales, las desigualdades sociales se mueven en órdenes de magnitud que resultan muy poco intuitivos, ya que muchas veces suponen pequeñas décimas o desviaciones frente a valores centrales o medios pero que pueden llegar a suponer grandes asimetrías. Incluso las personas con conocimientos matemáticos se enfrentan a sesgos cognitivos graves y sistemáticos tanto por lo que toca a la comprensión de realidades probabilísticas sencillas pero antiintuitivas (Palmarini, 2005) como a la evaluación de procesos en los que se dan enormes desniveles de magnitud alejados de los que manejamos en nuestra vida cotidiana, como las desigualdades de renta extremas. Y otro tanto ocurre con la velocidad a la que las desigualdades se desarrollan: suelen ser procesos a medio y largo plazo cuya correcta visualización es incompatible con las herramientas habituales.

Por eso, a la hora de examinar la actual crisis española desde el punto de vista de la desigualdad puede tener más sentido tomar en consideración el número de desahucios diarios, la tasa de familias con todos los miembros en paro o el descenso de consumo de ciertos productos que utilizar el índice de Gini. Y, sobre todo, parece muy recomendable emplear herramientas de visualización poco ortodoxas, como el desfile de los salarios, que permiten una percepción intuitiva de la magnitud real de las desigualdades (Shapiro y Friedman, 2006, gráfico 1).

El desfile de los salarios (income parade) es un experimento mental desarrollado por el economista holandés Jan Pen para proporcionar una imagen intuitiva de la magnitud de la desigualdad económica (Pen, 1971: 48 y ss). Consiste en suponer que la altura de cada habitante de un país es proporcional a sus ingresos, de modo que la gente pobre será muy baja y la gente rica muy alta. A continuación, imaginamos que todos ellos desfilan en una larga hilera, ordenados de menor a mayor tamaño, durante una hora. En el caso español, si suponemos una altura media de un metro y setenta centímetros, equivalente al salario medio bruto en 2010, el consejero delegado del Banco Santander mediría 750 metros y el empresario Florentino Pérez cinco kilómetros. También existen versiones del desfile aplicadas al patrimonio y que hacen visibles desigualdades aún mayores. 


\section{GRÁFICO 1:}

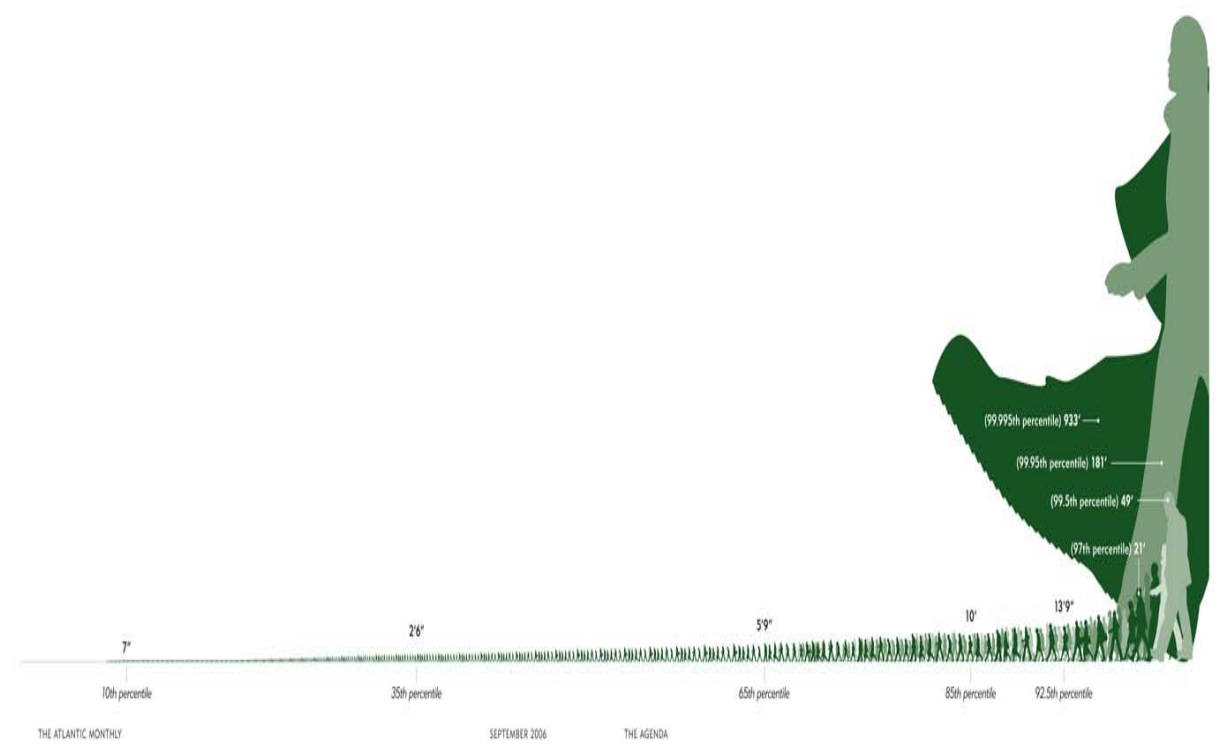

Fuente: “The Height of Inequality” (2006) The Atlantic,

http://www.theatlantic.com/magazine/archive/2006/09/the-height-of-inequality/305089/

\section{Conclusiones}

A modo de conclusión cabría apuntar que aunque los problemas relacionados con las desigualdades sociales fueron cruciales en los albores de la teoría social y aún son esenciales en la actualidad, seguimos sin disponer de herramientas de medición sencillas y precisas para analizarlas con fiabilidad. En concreto, en la actualidad, los debates sobre el crecimiento de las desigualdades sociales son de primera magnitud y la necesidad de proveer de datos sociológicos útiles se hace urgente. La mayoría de los indicadores heredados proceden de disciplinas circundantes a la sociología y, o bien no cumplen criterios matemático-estadísticos importantes, o bien no tienen utilidad sociológica al resultar ininterpretables según teorías sociales al uso, o bien no proporcionan imágenes realistas de las situaciones vividas o, incluso, carecen de fuentes de datos suficientes y completas. Es decir, si se intenta operacionalizar un concepto tan profundamente sociológico como el de desigualdad se corre el riesgo de caer por la pendiente resbaladiza del ultraformalismo, la falta de empiria o ausencia de datos, los laberintos estadísticos o las medidas sin teoría. La historia reciente de los denodados esfuerzos (y sus 
constantes matices, correcciones y reformulaciones) por traducir estas nociones (desigualdad, pobreza, privación, desarrollo, etc.) en cifras e indicadores medibles demuestra la complejidad de la tarea.

No obstante, si aceptamos estas limitaciones como constitutivas de este campo de estudio, podemos evitar algunos caminos cegados en la medición de la estratificación y avanzar por otros más prometedores y viables para la sociología. En particular, como aportación final de este artículo pensamos que la medición y el análisis de las desigualdades sociales deberían pasar por una serie de requisitos o condiciones a tener en cuenta:

i) Asumir una vinculación teórico-práctica adaptada al contexto histórico concreto que tenga en cuenta un concepto amplio de las clases sociales actuales (no reducida a la renta per cápita declarada o al salario bruto individual). De esta forma se podrían incluir otras variables que reflejen situaciones particulares e históricas e incluso medidas inversas o alternativas que puedan informar de los procesos sociales en curso. Las operacionalizaciones que se realicen deberían tener en cuenta un marco teórico ampliado que incorpore la coyuntura de los casos que se estudian.

ii) Elaborar un enfoque temporalmente amplio que tenga en cuenta el ritmo real de los modelos de acumulación socioeconómica existentes. La ampliación de las escalas temporales facilitaría la observación de fenómenos cuya sedimentación y consecuencias se produce en el medio plazo, casi siempre a lo largo de décadas. Las medidas fotográficas que facilitan muchos indicadores compuestos hacen perder (y no comprender) la dimensión histórica de los procesos sociales mucho más dependientes de desarrollos temporales amplios que de acontecimientos puntuales.

iii) Otorgar más importancia a las prácticas de visibilización y representación que al cómputo exacto, promoviendo una visión relativa y de contraste de las desigualdades sociales como un fenómeno global y estructural antes que como un mero dato. Las desigualdades en el capitalismo tardío son el fruto de procesos multicausales complejos cuya comprensión requiere herramientas de representación visual y gráfica ambiciosas. Justamente, dada la complejidad de la medición instrumental de dinámicas socioeconómicas profundas e históricas, la obsesión con cifras concretas oscurece y sombrea la naturaleza diacrónica de dichos fenómenos de amplio rango cuya interpretación sociológica gana al representarse visualmente. 


\section{Bibliografía}

Álvarez-Uría, F.; Varela, J. (2011). Sociología, capitalismo y democracia. Madrid: Morata.

Arriba González de Durana, A. (2002). El concepto de exclusión en política social. Documento de Trabajo, CSIC (Unidad de Políticas Comparadas), ${ }^{\circ} 1$.

Atkinson, A.B. (1970). On the measurement of inequality. Journal of Economic Theory, 3, 244-263.

Blalock, H. (1991). Understanding social inequality: Modeling allocation processes. Londres: Sage.

Brady, D. (2003). Rethinking the Sociological Measurement of Poverty, Social Forces 81 (3): 715-751.

Chen, S.; Ravallon, M. (2013). More relatively-poor people in a less absolutelypoor world, Review of Income and Wealth, 59(1), 1-28. DOI: 10.1111/j.14754991.2012.00520.x

Chossudowsky, M. (2002). Globalización de la pobreza y nuevo orden mundial. Madrid: Siglo XXI

CEPAL (2001). Consideraciones sobre el índice de Gini para medir la concentración del ingreso, Serie de estudios estadísticos y prospectivos CEPAL, $\mathrm{n}^{\circ}$ 9. Marzo, 2001.

Cowell, F. (2009). Measuring Inequality, LSE Perspectives in Economic Analysis. Oxford: Oxford University Press.

Del Río, A. (2008). El endeudamiento de los hogares españoles, Servicio de Estudios del Banco de España, Documento de Trabajo n. ${ }^{\circ}$ 0228. Madrid: Banco de España.

Dubet, F. (2006). Las desigualdades multiplicadas. Toluca: UAEM.

Duclos, J.Y.; Araar, A. (2006). Poverty and Equity: Measurement, Policy and Estimation with $D A D$, Otawa: Springer.

EAPN (2012). Las cifras de la pobreza y exclusión social en España de cara a Europa 2020, European AntiPoverty Network, http://www.eapn.es/ARCHIVO/documentos/recursos/1/1030_pobreza_exclusion espana.pdf

Escobar, M. (1998). Desviación, desigualdad, polarización: medidas de la diversidad social, Revista Española de Investigaciones Sociológicas nº 82, 9-36. 
Fernández Durán, R. (2011). La quiebra del capitalismo global 2000-2030. Barcelona: Virus.

Foessa (2013). Informe 2013. Desigualdad y Derechos Sociales. Madrid: Cáritas.

Freeland, C. (2012). Plutocrats: The Rise of the New Global Super-Rich and the Fall of Everyone Else. New York: Penguin Press.

Garzón Espinosa, A. (2013). La transición en el modelo de crecimiento de la economía española, Revista de Economía Crítica, nº 15, primer semestre, 74-79.

Graham, C. (2002). Mobility, opportunity and vulnerability: the dynamics of poverty and inequality in a Global Economy. Journal of Human Development 3(1), 57-94.

Goerlich, F. (1998). Desigualdad, diversidad y convergencia: (algunos) instrumentos de medida. Valencia: IVIE.

Hernández Pedreño, M. (2008). Pobreza y exclusión en las sociedades del conocimiento. En Hernádez Pedreño, M. (Coord.), Exclusión social y desigualdad. Murcia: Universidad de Murcia.

Hernández Vigueras, J. (2012). El casino que nos gobierna. Madrid: Clave Intelectual.

Heshmati, A. (2004). Inequalities and their measurement. Forschungsintitut zur Zukunft der Arbeit Institute for the Study of Labor, IZA, Julio 2004.

Instituto Nacional de Estadística (2004). Estudio descriptivo de la pobreza en España, accesible en http://www.ine.es/daco/daco42/sociales/estudiodesc.pdf

López, I.; Rodríguez, E. (2011). The Spanish Model. New Left Review 69, MayoJunio 2011, 5-29.

Manheim, J.; Rich, R. (1988). Análisis Político Empírico. Métodos de Investigación en Ciencia Política. Madrid: Alianza Editorial.

McKay, A. (2002). Defining and measuring inequality, Inequality Briefing Paper $\mathrm{n}^{\circ}$ 1, March 2002, DFIF\&ERC.

Milanovic, B. (2012). Los que tienen y los que no tienen. Una breve historia de la desigualdad global. Madrid: Alianza.

Navarro, V. (2009). El subdesarrollo social de España. Madrid: Alianza.

Pen, J. (1971). Income distribution. Londres: Penguin. 
Piatelli Palmarini, M. (2005). Túneles de la mente. Barcelona: Crítica.

Piketty, T.; Saez, E. (2006). The Evolution of Top Incomes: A Historical and International Perspective, American Economic Review: Papers and Proceedings, 96, 2 (May 2006): 200-205.

Sen, A.; Foster, J. (1996). On Economic Inequality. Oxford: Oxford University Press.

Subirats, J. (Dir.) (2005). Análisis de los factores de exclusión social. Madrid: Fundación BBVA, en http://w3.grupobbva.com/TLFBBindex_pub.jsp

Tezanos, J.F. (1999). Tendencias de dualización y exclusión social en las sociedades avanzadas. Un marco para el análisis. En J. F. Tezanos (Ed.), Tendencias en desigualdad y exclusión social. Tercer foro sobre tendencias sociales. Madrid: Sistema.

Townsend, P.; Phillimore, P.; Beattie, A. (1988). Health and Deprivation: Inequality and the North. London: Routledge.

UUNN (2010). Innovaciones en la medición de la desigualdad y la pobreza, cap. 5, Informe Desarrollo Humano 2010: http://www.hdr.undp.org/en/.../HDR_2010_ES_Chapter5_reprint.pdf

Urban, I. (2013). Implementation Issues in the Duclos-Jalbert-Araar Decomposition of Redistributive Effect, Public Finance Review, 41: 121-143. DOI: $10.1177 / 1091142112452777$.

Vakis, R.; Schmidt, M. (2010). Innovación en la medición de pobreza: Siete lecciones de diseño sobre el uso de Comités Asesores para mejorar las cifras oficiales de pobreza en Perú, En Breve, Banco Mundial, n ${ }^{\circ} 155$. http://siteresources.worldbank.org/INTLAC/Resources/2578031269390034020/EnBreve_155_Spanish_Web.pdf

Wallerstein, I. (2001). El capitalismo histórico. Madrid: Siglo XXI.

Wilkinson, R.; Pickett, K. (2009). Desigualdad. Un análisis de la infelicidad colectiva. Madrid: Turner. 